\title{
Comparaison des politiques municipales de Saguenay et Sherbrooke en matière d'attractivité urbaine
}

\author{
Chantale Munger \\ Cégep de Jonquière
}

\section{INTRODUCTION}

\section{L'objectif de cette recherche est de comparer les politiques municipales de deux villes moyennes québécoises, soit Saguenay et Sherbrooke.}

Dans le contexte de la mondialisation où tous les territoires sont en concurrence pour attirer les capitaux et les individus, les métropoles et les grandes villes détiennent une position de choix. Au Québec, les régions métropolitaines de Montréal et surtout de Québec tirent bien leur épingle du jeu. Toutefois, il en est autrement des villes et régions périphériques qui vivent parfois des problématiques inquiétantes sur les plans démographique et économique. Afin de renverser la situation, les gouvernements locaux choisissent fréquemment d'adopter des politiques familiales ou des stratégies reliées à l'immigration, notamment au sein des villes moyennes. On tente ainsi de rendre les territoires attractifs pour les ménages, les nouveaux arrivants et les entreprises.
Les villes moyennes constituent des espaces socioéconomiques intermédiaires situés entre les milieux ruraux et les grandes agglomérations urbaines. Celles-ci ont un rôle significatif à jouer dans le développement territorial. En conséquence, l'objectif de cette recherche est de comparer les politiques municipales de deux villes moyennes québécoises, soit Saguenay et Sherbrooke. L'analyse de contenu a porté sur la comparaison des documents municipaux en ce qui a trait aux efforts d'attractivité urbaine. À l'intérieur de ce texte, nous discuterons d'abord du contexte démographique des milieux périphériques avant de traiter du rôle des villes moyennes dans le champ du développement. En troisième lieu, nous résumerons les démarches méthodologiques. Finalement, les grands constats de la démarche comparative seront énoncés, révélant la situation avantageuse de Sherbrooke à plusieurs égards.

\section{LE CONTEXTE DÉMOGRAPHIQUE DIFFÉRENCIÉ DES TERRITOIRES}

\section{Les politiques natalistes des gouvernements supérieurs ont permis d'améliorer les profils démographiques de nombre de milieux.}

Dans l'ensemble du Québec, les indicateurs de fécondité ont diminué au cours des quarante dernières années: faible taux de natalité, vieillissement de la population, etc. D'ailleurs, l'indice synthétique de fécondité y est l'un des plus bas au Canada malgré une remontée récente (1.69 en 2011). Le groupe de recherche de Cirano ${ }^{1}$ prévoit qu'entre 2011 et 2031, le Québec connaîtra une baisse de $13 \%$ des 15 à 64 ans et une augmentation de $15 \%$ des 65 ans et plus. Les inégalités interrégionales accroissent ces problèmes dans les villes et régions localisées à l'extérieur de la plaine du Saint-Laurent. Ainsi, certaines municipalités ont connu une croissance de la population de plus de $30 \%$ (Blainville : $46 \%$ et Terrebonne : $31 \%$ ) entre 1996 et 2014 alors que certaines localités stagnent ou déclinent (Saguenay : $-6 \%)^{2}$. Ces conditions qui prévalent depuis quelques décennies déjà auront un impact négatif sur le renouvellement des générations, le remplacement de la main-d'œuvre, le fardeau fiscal des contribuables et l'occupation des territoires. Les politiques natalistes des gouvernements supérieurs ont permis d'améliorer 
les profils démographiques de nombre de milieux (allocations familiales, crédits d'impôt, CPE à faibles coûts, etc.). Cependant, les taux de natalité continuent de demeurer insuffisants pour favoriser la croissance.

Des politiques d'immigration ont donc été mises en place à l'échelle municipale afin de soutenir l'effort de pérennisation des territoires. Selon Long et
Légaré $^{3}$, "l'immigration atténue le déclin de la population et de la main-d'œuvre et fait diminuer la proportion des personnes âgées, le rapport de dépendance total et le rapport de dépendance des personnes âgées ». Cependant, les grands centres accueillent la plus grande proportion d'immigrants au pays ${ }^{4}$. En ce sens, la régionalisation de l'immigration est essentielle pour soutenir les efforts endogènes des territoires périphériques.

\section{VILLES MOYENNES, ATTRACTIVITÉ URBAINE ET DÉVELOPPEMENT}

Dans le contexte de la mondialisation, les villes de différentes tailles forment des réseaux d'échanges hiérarchisés. Les métropoles constituent les points forts de l'espace géographique. Celles-ci drainent les cadres les plus recherchés, les bourses financières de même que les sièges des grandes organisations internationales. D'autres pôles de moindre importance se greffent à ce système, soit les villes moyennes. Plus précisément, ces villes apparaissent comme des relais entre le local et le mondial ${ }^{5}$. Elles sont souvent un moteur économique et un pôle de services pour les territoires qu'elles desservent ${ }^{6}$.

Cependant, les seuils permettant de définir les villes moyennes ne font pas l'unanimité. Il ne s'agit donc pas de statuer sur un seuil absolu, mais sur leur poids relatif. Elles se définissent plus par la place qu'elles occupent et le rôle qu'elles jouent entre les grandes agglomérations et le rural $^{7}$. La ville moyenne participe ainsi au développement régional grâce à ses fonctions d'intermédiation pour les populations environnantes. Les territoires collaborent parfois et sont en compétition la plupart du temps. S'ils veulent conserver un avantage comparatif, ils doivent demeurer attractifs autant pour les membres de leur communauté que pour les entrepreneurs ou les futurs émigrants.

L'attractivité se définit par la «...capacité pour un territoire d'offrir aux acteurs des conditions qui les convainquent de localiser leurs projets sur leur territoire plutôt que sur un autre $»^{8}$. Certains territoires sortent gagnants de cette compétition, alors que d'autres perdent au change. Pour Ingallina ${ }^{9}$, le territoire devient le centre des stratégies, la composante première du changement et la source des représentations. L'attractivité territoriale est un concept relatif, il n'existe pas de recettes magiques permettant d'y arriver. Les qualités attractives d'un milieu peuvent différer d'un lieu à l'autre et d'un individu à l'autre. Il est cependant possible de réaliser un classement en grandes catégories des facteurs intervenants dans l'attractivité d'un territoire à partir de recherches et théories portant sur le sujet.

Des chercheurs comme Ingallina, Borja, Hatem ou Thiard ont permis de mieux comprendre le pouvoir de l'attractivité. Pour certains, il s'agit des facteurs liés au sentiment d'appartenance ${ }^{10}$, aux conditions de vie, de travail et d'accessibilité aux services ${ }^{11}$ ou encore au développement des emplois, des services publics, de la qualité des logements ou des espaces publics $^{12}$. De même, la création de pôles économiques $^{13}$ et innovateurs permettrait d'attirer les investisseurs et créer les emplois afin de retenir les ménages. Le développement du marketing municipal dans certaines municipalités constitue un effort de valorisation des territoires permettant d'insérer le local dans le processus de la mondialisation. Il peut servir à mettre en valeur ses avantages comparatifs ${ }^{14}$ et donner du sens au territoire ${ }^{15}$. Pour d'autres, l'attractivité est liée à l'innovation, à la qualité de la gouvernance locale et à la réactivité des acteurs locaux face aux attentes des entreprises ${ }^{16}$.

\section{Le développement du marketing municipal dans certaines municipalités constitue un effort de valorisation des territoires permettant d'insérer le local dans le processus de la mondialisation.}

La thèse élaborée par Florida ${ }^{17}$ consiste à créer une classe créative en attirant une classe sociale particulière dans les centres urbains. Cette classe d'individus créatifs faisant partie des artistes, 
créateurs, innovateurs liés aux arts, à l'éducation et aux sciences permettrait de créer un climat social attractif générateur d'un dynamisme innovateur. Le gouvernement du Québec publiait en 2011 sa Stratégie pour assurer l'occupation et la vitalité des territoires 2011-2016. Dans ce document, le sentiment d'appartenance, la cohabitation harmo-

\section{MÉTHODOLOGIE ET ÉTUDES DE CAS}

\section{Des grilles d'analyse ont permis de comparer les schémas d'aménagement et de développement, les plans stratégiques, les politiques d'immigration, les politiques culturelles, sociales et familiales communs aux deux villes.}

Le choix des villes moyennes s'est porté sur Saguenay et Sherbrooke, deux zones urbaines dotées d'une population totale d'ampleur comparable $^{19}$. Elles tiennent le rôle de capitale régionale, subissent l'influence internationale et nationale tout en ayant un impact sur le dynamisme régional ${ }^{20}$. Néanmoins, l'une de celles-ci est située à l'intérieur d'un rayon de $150 \mathrm{~km}$ de la métropole montréalaise pendant que l'autre est positionnée en périphérie de l'écoumène québécois. De plus, Sherbrooke a connu une croissance de population de $15 \%$ entre 1996 et 2013 alors que Saguenay subissait des pertes de $6 \%$ sur la même période ${ }^{21}$. De même manière, de 2001 à 2013, le solde des migrations internationales et interprovinciales pour Sherbrooke s'est établi à 12951 personnes contre 53 personnes pour Saguenay ${ }^{22}$. Afin de mesurer les efforts d'attractivité consentis par les deux villes, nous avons choisi de comparer les politiques publiques de même que le plan stratégique et le schéma d'aménagement et de développement qu'elles ont adoptés.

Selon le MAMROT ${ }^{23}$, la planification stratégique doit conduire à un mieux-être collectif tout en se distinguant des collectivités voisines. Celle-ci doit établir un diagnostic des conditions internes et externes, préciser les besoins des populations, dégager une vision d'avenir et définir les options stratégiques et les résultats attendus avant d'élaborer un plan d'action global. Les politiques publiques constituent un ensemble de mesures concrètes $^{24}$ et représentent pour Dye ${ }^{25}$ « tout ce que les gouvernements choisissent de faire ou ne pas nieuse avec les communautés allochtones et autochtones, la mixité sociale, culturelle et intergénérationnelle, l'accessibilité et la qualité de vie, la densification urbaine, l'écoresponsabilité, la mise en valeur du patrimoine culturel et naturel font partie des dix-neuf objectifs liés aux axes d'intervention proposés ${ }^{18}$.

faire ». L'analyse de ces politiques permet au chercheur de considérer un ou plusieurs aspects à la fois, de dégager les enjeux et d'obtenir à partir des représentations sous-jacentes une vision des objectifs poursuivis ${ }^{26}$.

Des grilles d'analyse ont permis de comparer les schémas d'aménagement et de développement, les plans stratégiques, les politiques d'immigration, les politiques culturelles, sociales et familiales communs aux deux villes. Les variables retenues pour construire les grilles d'analyse proviennent de l'analyse des relevés de littérature, grâce aux concepts mis de l'avant dans la théorie de la classe créative, dans les concepts liés à la vitalité des territoires du gouvernement québécois et des auteurs provenant de divers horizons liés à la sociologie, à la géographie ou à l'économie. Pour nommer quelques concepts retenus en raison de leur importance selon les chercheurs: sentiment d'appartenance, marketing, identité, accessibilité, emplois, culture, tolérance, diversité, communication, réseautage, pôles, concertation, éducation ou environnement.

La première étape consiste à calculer les fréquences des concepts-clés dans les politiques en soulignant l'importance relative de ces concepts. Par exemple à Sherbrooke, la culture, la mobilité liée aux moyens de transport, les paysages, l'environnement, de même que la concertation dominent le classement. Bien que les loisirs, l'accessibilité, la créativité, le patrimoine, la diversité, l'intégration sociale et l'innovation soient indiqués dans les textes plus d'une centaine de fois. À Saguenay, la culture et la diversité sont les deux seuls concepts qui apparaissent plus d'une centaine de fois dans les documents. En effet, les politiques sherbrookoises (2856 fois) utilisent plus souvent les thèmes à l'étude que celles de Saguenay (1259). Dans le cas de Sherbrooke, c'est dans le schéma d'aména- 
gement et de développement que l'on retrouve la plus grande fréquence des concepts (1189), alors que pour Saguenay c'est dans la politique culturelle (488).

En fait, pour chaque document domine un ou deux concepts. Pour Saguenay, la vision stratégique met l'accent sur la fabrication de créneaux économiques de même que sur les paysages. Dans le schéma d'aménagement et de développement, c'est la mobilité et les paysages qui dominent. Dans la politique familiale : l'accessibilité et la sécurité sont les mots-clés tandis que la politique sociale vise l'équité et la concertation. La politique d'immigration s'appuie sur les concepts d'immigration et d'intégration. Quant à la politique culturelle, la culture et le patrimoine sont les termes les plus fréquents. Dans le cas de Sherbrooke, la vision stratégique de même que le schéma d'aménagement et de développement soulignent l'importance des paysages et de la mobilité. La concertation est le concept fort des politiques familiale et sociale. La communication est le deuxième concept de la politique familiale alors que la culture est le second terme de la politique sociale. La politique d'immigration, à l'instar de Saguenay s'appuie aussi sur l'immigration et l'intégration. Quant à la politique culturelle, le terme principal est sans surprise la culture suivi par celui d'artistes.

\section{COMPARAISON DES POLITIQUES MUNICIPALES}

\section{Sur la quarantaine de critères soulevés dans les recherches portant sur l'attractivité et leur recension dans les différentes politiques publiques des deux villes à l'étude, dix catégories-synthèse permettent de rendre compte de l'analyse.}

Les politiques des deux villes abordent chaque orientation $^{27} \mathrm{du}$ document du gouvernement $\mathrm{du}$ Québec concernant l'occupation et la vitalité des territoires $^{28}$ à l'exception des aspects ayant trait aux relations avec les autochtones, à la maind'œuvre qualifiée et à la décentralisation. Alors que Saguenay cible le retour des jeunes, Sherbrooke n'en fait aucune mention. Saguenay reprend les termes de culture, arts, patrimoine, tolérance et ouverture aux immigrants chers à la théorie des classes créatives. Sherbrooke utilise plus largement l'ensemble des concepts retenus de cette théorie. Les concepts déjà énumérés à Saguenay, tel que culture, arts, patrimoine ou tolérance aux immigrants sont utilisés, mais aussi des concepts propres à la théorie tels que « authenticité », « ambiance » ou « night life ».

Sur la quarantaine de critères soulevés dans les recherches portant sur l'attractivité et leur recension dans les différentes politiques publiques des deux villes à l'étude, dix catégories-synthèse permettent de rendre compte de l'analyse. Le concept le plus important pour les deux villes est celui de culture auquel nous avons associé les termes d'artistes, de patrimoine et d'animation culturelle. Dans les documents de Saguenay, cette catégorie apparait 312 fois et plus du double pour Sherbrooke (721 fois). Les aspects liés aux conditions de vie (logement, loisirs, environnement...) sont utilisés 658 fois à Sherbrooke et 137 fois à Saguenay. L'accessibilité (mobilité, densification urbaine) bien que très importante à Sherbrooke (328 fois) est en deuxième position à Saguenay (147 fois). La compétition (216 fois), la concertation (212 fois), et l'ouverture (tolérance, intégration : 165 fois) représentent pour Sherbrooke des critères importants d'attractivité. À Saguenay, l'ouverture apparait en quatrième position (111 fois) alors que la compétition (pôle de développement : 69 fois) et la concertation (65 fois) sont utilisées une soixantaine de fois dans les documents. Au contraire, à Saguenay, les concepts liés à l'image, au sentiment d'appartenance, à la communication ou au réseautage sont les moins utilisés. Les quatre concepts retiennent l'attention pour la même raison à Sherbrooke dans des proportions semblables. Dans le cas des deux villes, les populations ciblées par les politiques d'attraction sont les étudiants, les professeurs et les chercheurs. Les villes aspirent aussi à attirer les jeunes ménages et les populations issues de l'immigration surtout francophones. 
Tableau 1 - Fréquence des catégories de concepts liés à l'attraction urbaine : Saguenay et Sherbrooke

\begin{tabular}{|l|l|l|}
\hline Concepts & Saguenay & Sherbrooke \\
\hline Culture (artistes, patrimoine, animation) & 312 & 721 \\
\hline $\begin{array}{l}\text { Conditions de vie (logement, loisirs, } \\
\text { environnement...) }\end{array}$ & 137 & 658 \\
\hline Accessibilité, (mobilité, densification) & 147 & 328 \\
\hline Compétitif, innovateur (pôle...) & 69 & 216 \\
\hline Ouverture, tolérance, intégration & 111 & 165 \\
\hline Concertation & 65 & 212 \\
\hline Image (marketing) & 22 & 8 \\
\hline Sentiment d'appartenance (identité) & 21 & 27 \\
\hline Communication (information) & 24 & 44 \\
\hline Réseaux & 2 & 4 \\
\hline
\end{tabular}

Les tableaux 2 et 3 indiquent la présence ou l'absence des catégories choisies. Par exemple, à Saguenay la présence des termes « conditions de vie » et «concertation » apparaissent dans presque tous les documents alors que le réseautage n'apparait que dans le schéma d'aménagement et de développement et la vision stratégique. La politique sociale fait intervenir deux concepts : concertation et conditions de vie.

Tableau 2 - Présence des concepts-clés de l’attractivité des territoires : Saguenay

\begin{tabular}{|c|c|c|c|c|c|c|c|c|c|}
\hline Politique & 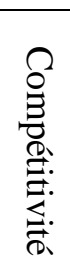 & 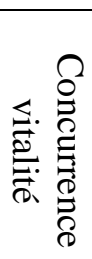 & 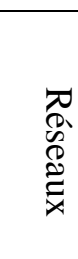 & 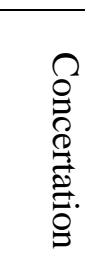 & $\begin{array}{l}\stackrel{\gtrless}{\Xi} \\
\stackrel{\Xi}{\Xi} \\
\stackrel{\Xi}{\Xi}\end{array}$ & 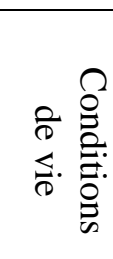 & 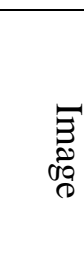 & 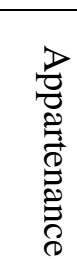 & 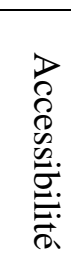 \\
\hline Vision & $X$ & $X$ & $X$ & $X$ & $X$ & $X$ & $X$ & & \\
\hline Schéma & $X$ & & $X$ & & $X$ & $X$ & $X$ & $X$ & \\
\hline Famille & $X$ & $X$ & & $X$ & $X$ & $X$ & $X$ & & $X$ \\
\hline Culture & $X$ & $X$ & & $X$ & & $X$ & $X$ & $X$ & $X$ \\
\hline Immigr & & $X$ & & $X$ & $X$ & $\mathrm{X}$ & & $\mathrm{X}$ & $X$ \\
\hline Sociale & & & & $X$ & & $X$ & & & \\
\hline
\end{tabular}

Pour Sherbrooke, les concepts de «conditions de vie », «concertation» et «accessibilité » se retrouvent dans la quasi-totalité des documents à l'étude. Le concept d'image n'a été indiqué que dans la vision stratégique de la ville. Chacune des politiques aborde au moins cinq ou six concepts. 


\begin{tabular}{|c|c|c|c|c|c|c|c|c|c|}
\hline Politique & 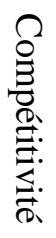 & 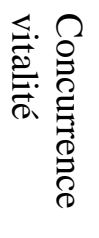 & 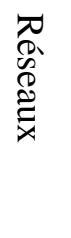 & 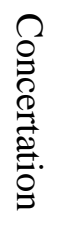 & 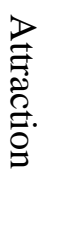 & 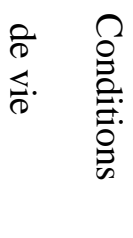 & 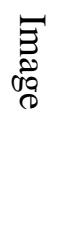 & 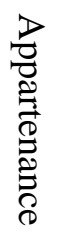 & 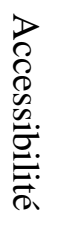 \\
\hline Vision & $X$ & $X$ & $X$ & $X$ & $X$ & $\mathrm{X}$ & $X$ & $X$ & $\mathrm{X}$ \\
\hline Schéma & $X$ & $X$ & $X$ & $X$ & $\mathrm{X}$ & $\mathrm{X}$ & & & \\
\hline Familiale & & $X$ & & $\mathrm{X}$ & $X$ & $\mathrm{X}$ & & $X$ & $\mathrm{X}$ \\
\hline Culture & $X$ & $X$ & $X$ & $X$ & & $X$ & & $X$ & $\mathrm{X}$ \\
\hline Immigr & & & & $X$ & $X$ & $X$ & & $X$ & $\mathrm{X}$ \\
\hline Sociale & $X$ & & $X$ & $X$ & $X$ & $X$ & & $\mathrm{X}$ & $X$ \\
\hline
\end{tabular}

\section{CONCLUSION}

Dans les deux cas, les villes désirent valoriser la qualité de vie sur leur territoire, grâce à une population stable ou en augmentation, une économie prospère et innovatrice, un environnement naturel sain et un milieu de vie dynamique et attrayant.

L'attractivité des territoires est un projet à renouveler sans cesse et chaque ville moyenne doit trouver sa propre voie dans les réseaux nationaux et internationaux. Les résultats de cette recherche ont montré de nombreuses ressemblances entre les deux villes. Il ressort des politiques que les deux villes désirent contribuer au bien-être et à la prospérité de leur population. La différence tient à la façon de contribuer à ce bien-être. Dans le cas de Saguenay, c'est en stabilisant la population et en s'adaptant à son vieillissement, en empruntant la voie économique, la formation et la recherche ainsi que par l'immigration. En économie, c'est par la création de créneaux, par le partenariat, la création de la cité du savoir, le développement des industries touristiques et agricoles et l'amélioration des transports. Sherbrooke met l'accent sur l'ambiance, les espaces de détentes, des centres-villes dynamiques, la mise en valeur du patrimoine et une offre de services culturels et sportifs. Pour l'aspect économique, Sherbrooke veut développer l'économie du savoir et consolider sa base économique. Elle tient à offrir des milieux de vie et un environnement de qualité tout en favorisant l'innovation sociale et la participation à la vie communautaire. Dans les deux cas, les villes désirent valoriser la qualité de vie sur leur territoire, grâce à une population stable ou en augmentation, une économie prospère et innovatrice, un environnement naturel sain et un milieu de vie dynamique et attrayant. Par ailleurs, certains aspects comme le réseautage, la communication ou encore le sentiment d'appartenance auraient avantage à être renforcés. Pour faire suite à cette analyse, il serait pertinent de confronter les orientations émises dans les documents politiques avec les décisions et comportements adoptés par les élus de ces deux municipalités. Y-a-t-il concordance entre l'écrit et la gestion quotidienne?

\section{BIBLIOGRAPHIE}

Borja, Jordi (2007). "L'attractivité : les conditions locales de la réussite globale » in Ministère de l'Écologie, de l'Énergie, du Développement durable et de l'Aménagement du territoire. Actes des séminaires 2007, L'attractivité des territoires. Regards croisés. [En ligne] consulté le 25 mars 2014, URL : http://rp.urbanisme.equipement.gouv.fr/puca/ edito/actes_attr_territoires.pdf

Cavallier, Georges, 2002, "Les villes, acteurs de la mondialisation », Ramses [en ligne], 13 mars 2014, http://www.ifri.org/files/Ramses2002/R02_CAVALL IER.pdf 
Chanoux Maeva, (2013). La rencontre entre la marque et le territoire: intégration du concept de marque dans les pratiques de marketing territorial, dir. Robert Fouchet \& Olivier Keramidas, IMPGT, Aix-Marseille Université.

CIRANO (2013) «Le Québec économique» [en ligne], le 20 novembre 2013, qe.cirano.qc.ca/tab/ deces/indice_synthetique_de_fecondite_Quebec Charbonneau, François, Lewis, Paul, Manzagol, Claude (2003). Villes moyennes et mondialisation. Renouvellement de l'analyse et des stratégies. Université de Montréal, Trames, 334 p.

DI MÉO, GUY (2010). « La métropolisation. Une clé de lecture de l'organisation contemporaine des espaces géographiques ». L'information géographique, no 3 , p. $23-38$

Dye, Thomas R, Understanding Public Policy, Englewood Cliffs, Prentice Hall, 1978, p. 3-4

Florida, Richard (2002). The Rise of the creative class. Why cities without gays and rock bands are losing the economic development race.

Gouvernement du Québec (2011) « Nos territoires : y habiter et en vivre ». Stratégie pour assurer l'occupation et la vitalité des territoires 2011-2016, 72 p. [en ligne] http://www.mamrot.gouv.qc.ca/ pub/occupation_territoire/strategie_occupation.pdf, consulté le 9 février 2015

Gouvernement du Québec (2013). « Présence en 2013 des immigrants admis au Québec de 2002 à 2011 ». [en ligne], http://www.midi.gouv. qc.ca/publications/ fr/recherches-

statistiques/PUB_Presence2013_admisQc_02_11.pdf, consulté le 10 février 2015

HATEM, Fabrice (2007). « Le rôle des clusters dans les politiques d'attractivité » in Ministère de l'Écologie, de l'Énergie, du Développement Durable et de l'Aménagement du territoire. Actes des séminaires 2007, «1'attractivité des territoires ». Regards croisés. [En ligne] consulté le 25 mars 2014, URL : http://rp.urbanisme.equipement.gouv.fr/puca/edito/act es_attr_territoires.pdf

Ingallina, Patricia (2007). «L'attractivité des territoires », in Ministère de l'Écologie, de l'Énergie, du Développement Durable et de l'Aménagement du territoire. Actes des séminaires 2007, l'attractivité des territoires. Regards croisés. [En ligne] consulté le 25 mars 2014, URL : http://rp.urbanisme.equipement. gouv.fr/puca/edito/actes_attr_territoires.pdf Institut de la statistique du Québec (2014a) Estimation de la population [en ligne], le 13 mars 2014 http://www.stat.gouv.qc.ca/statistiques/populationdemographie/structure/mun 15000.htm Institut de la statistique du Québec, Migrations internationales et interprovinciales [en ligne], le 15 mars 2014, http://www.stat.gouv.qc.ca/statistiques/ population-demographie/migration/internationalesinterprovinciales/index.html

Long, M, Légaré, Jacques (2003) « Les politiques de fécondité en Chine et d'immigration au Canada : étude comparée de leurs impacts sur le vieillissement », Cahiers québécois de démographie, vol. 32, no 1, p. 741.

Ministère des Affaires municipales, des régions et de l'occupation du territoire (2014) Planification stratégique, Outils de planification [En ligne], le 26 mars 2014, http://www.mamrot.gouv.qc.ca/ amenagement-du-territoire/guide-la-prise-de-decision -en-urbanisme/planification/planification-strategique/ Muller, Pierre (2011). Les politiques publiques, Que sais-je? puf, 126 p.

Muller, Pierre (2000). «L'analyse cognitive des politiques publiques : vers une sociologie politique de l'action publique », Revue française de science politique, volume 50, no 2, p. 189-208 [en ligne] consultée le 31 mars 2014, http://www.persee.fr/ web/revues/home/prescript/article/rfsp_0035-

2950 2000_num 50_2 395464

Porter, Michael (1993). L'avantage concurrentiel des nations, éd. InterEditions, Alternatives Économiques $\mathrm{n}^{\circ} 111$ - novembre 1993 [en ligne] http:// www.alternatives-economiques.fr/l-avantageconcurrentiel-des-nations-michael-porter_fr_art_66 6700.html consulté le 9 février 2015

Proulx, Marc-Urbain, Tremblay, Dominic (2006). « Marketing territorial et positionnement mondial », Lavoisier, Géographie, Économie, Société, vol 8, 239-256

Roncayolo, Marcel (2007). «Réflexions autour de la notion d'attractivité $\gg$. In Ministère de l'Écologie, de l'Énergie, du Développement Durable et de l'Aménagement du territoire. Actes des séminaires 2007, l'attractivité des territoires. Regards croisés. [En ligne] consulté le 25 mars 2014, URL: http://rp.urbanisme.equipement.gouv.fr/puca/edito/act es_attr_territoires.pdf

Saint-Julien, T. (2003), «Les villes moyennes en Europe, contextes et défis ». pp. 20-28 in Charbonneau, F., Lewis, P., Manzagol, C.(2003) Villes moyennes et mondialisation. Renouvellement de l'analyse et des stratégies. Université de Montréal, Trames, 334 p.

Thiard, Philippe (2007). «Attractivité et compétitivité : offre territoriale, approches marketing et retombées » In Ministère de l'Écologie, de l'Énergie, du Développement Durable et de l'Aménagement du territoire. Actes des séminaires 2007, l'attractivité des territoires. Regards croisés. [En ligne] consulté le 25 mars 2014, URL : http://rp.urbanisme.equipement. gouv.fr/puca/edito/actes_attr_territoires.pdf Wackermann, Gabriel (2000) Très grandes villes et métropolisation, ellipses, Capes/Agrégation, 223 p. 


\section{NOTES}

${ }^{1}$ CIRANO, 2013

${ }^{2}$ Institut de la statistique du Québec [en ligne], le 13 mars 2014, http://www.stat.gouv.qc.ca/statistiques/population-

demographie/structure/mun_15000.htm et calcul par l'auteure.

${ }^{3}$ Long, M, Légaré, J, 2003

${ }^{4}$ Montréal, immigration de 2001 à 2011 : $75.2 \%$ des immigrants encore présents au Québec en 2013. http://www.midi.gouv.qc.ca/publications/fr/recherchesstatistiques/PUB_Presence2013_admisQc_02_11.pdf

${ }^{5}$ Cavallier, G, 2002

${ }^{6}$ Gouvernement du Québec, $2011: 21$

${ }^{7}$ Saint-Julien, T, 2003

${ }^{8}$ Hatem, 2004

${ }^{9}$ Ingalina, 2007

${ }^{10}$ Ibid

${ }^{11}$ Roncoyolo, 2007

${ }^{12}$ Borja : 2007

${ }^{13}$ Porter : 2000, Hatem : 2007

${ }^{14}$ Proulx, Tremblay, 2006

${ }^{15}$ Chanoux,2013

${ }^{16}$ Thiard, $2007: 47$

${ }^{17}$ Florida, 2002

${ }^{18}$ Gouvernement du Québec: Stratégie pour assurer l'occupation et la vitalité des territoires 2011-2016.

${ }^{19}$ Population en 2013: Sherbrooke: 160745 , population Saguenay: 146 387. Gouvernement du Canada : www.stat.qc.ca

${ }^{20}$ Charbonneau et al. : 2003

${ }^{21}$ Institut de la statistique du Québec [en ligne], le 13 mars 2014, http://www.stat.gouv.qc.ca/statistiques/population-

demographie/structure/mun 15000.htm et calcul par l'auteure.

${ }^{22}$ Institut de la statistique du Québec [en ligne], le 15 mars 2014, http://www.stat.gouv.qc.ca/statistiques/population-

demographie/migration/internationales-

interprovinciales/index.html, agrégations de l'auteur.

${ }^{23}$ MAMROT, 2014

${ }^{24}$ Muller, 2011

${ }^{25}$ Dye, 2010

${ }^{26}$ Muller, 2000, P. 190

${ }^{27}$ Grandes orientations : culture, accessibilité et conditions de vie, infrastructure et communication, économie et finance. Ainsi les termes de patrimoine culturel, accueil, intégration, cohabitation harmonieuse, sentiment d'appartenance, accessibilité aux logements, mobilité durable ou collaboration ont tous été abordés.

${ }^{28}$ Gouvernement du Québec: Stratégie pour assurer l'occupation et la vitalité des territoires 2011-2016. 